\title{
MATRICES WITH A SEQUENCE OF ACCRETIVE POWERS
}

\author{
BY \\ DANIEL HERSHKOWITZ AND HANS SCHNEIDER* \\ Mathematics Department, University of Wisconsin, Madison. WI 53706, USA ; and \\ Department of Mathematics, Technion - Israel Institute of Technology, Haifa 320(K), Israel
}

ABSTRACT

It is shown that a matrix satisfying a certain spectral condition which has an infinite sequence of accretive powers is unitarily similar to the direct sum of a normal matrix and a nilpotent matrix. If the sequence of exponents is forcing or semiforcing then the spectral condition is automatically satisfied. If, further, the index of 0 as an eigenvalue of $A$ is at most 1 or the first term of the sequence of exponents is 1 , then the matrix is positive semidefinite or positive definite. There are applications to matrices with a sequence of powers that are $M$-matrices.

\section{§1. Introduction}

Let $A$ be a (complex $n \times n$ ) matrix. The matrix $A$ is called accretive if $\operatorname{Re}(A)=\left(A+A^{*}\right) / 2$ is a positive semidefinite (Hermitian) matrix $(\operatorname{Re}(A) \geqq 0)$ and $A$ is called strictly accretive if $\operatorname{Re}(A)$ is a positive definite matrix $(\operatorname{Re}(A)>$ $0)$. In $1975, \mathrm{C}$. R. Johnson [6] showed that if $A, A^{2}, A^{3}, \ldots$ are strictly accretive then $A$ is positive definite. A proof valid for bounded linear operators on a complex Hilbert space was given by DePrima and Richard in [2]. In fact they showed that if $A, A^{2}, A^{3}, \ldots$ are accretive then $A$ is positive semidefinite. In 1976 Shiu [9] improved the result of [2]. Basing his results on some theorems in Nagy-Foias [10, Chapter 4, Section 4], he proved the following theorem:

Let $r_{k}=2^{k-1}, k=1,2, \ldots$ If

$$
A^{r_{k}} \text { is accretive for } k=1,2, \ldots
$$

then $A \geqq 0$.

'The research of the second author was supported in part by NSF grant DMS-8320189, by ONR grant N00014-85-K-1613 and by the Lady Davis Foundation of Israel.

The first author is located permanently at the Technion and the second author at the University of Wisconsin.

Received August 5, 1985 and in revised form February 10, 1986 
In this paper we generalize the above results in the finite dimensional case. We employ the concept of a (semi)forcing sequence of positive integers $\left(r_{1}, r_{2}, \ldots\right)$ defined in our paper [5]. We show that if (1.1) holds for a semiforcing sequence and either the index of 0 as an eigenvalue of $A$ is at most 1 or $r_{1}=1$, then it follows that $A \geqq 0$. Further, if either the sequence is semiforcing, (1.1) holds and $A^{p}$ is strictly accretive for some postive $p$, or the sequence is semiforcing and

$$
A^{r_{k}} \text { is strictly accretive for } k=1,2, \ldots
$$

then $A>0$.

We use the above theorems to improve a recent result in [3]. There it is shown that a matrix all of whose positive powers are irreducible $M$-matrices is positively diagonally similar to a symmetric matrix. Here we derive the same conclusion from a weaker hypothesis, and then apply this result to show that all powers of the given matrix are irreducible $M$-matrices.

We now describe our results in more detail. In Section 2, which is purely number theoretical, we define the concept of a forcing [semiforcing] sequence (for a set of complex numbers), namely an increasing sequence of positive integers with the property that a complex number $c$ (in the set) is forced to be nonnegative if for every $p$ in the sequence $c^{p}$ has nonnegative [positive] real part, see Definition 2.1. We recall sufficient conditions for a sequence to be (semi)forcing proved in [5] (Theorems 2.5 and 2.8). Some examples of (semi)forcing sequences are given.

In Section 3 we prove our main results on matrices with accretive powers. Our first (and key) theorem (Theorem 3.1) asserts that a matrix which satisfies a certain spectral condition and which has an infinite sequence of accretive powers is unitarily similar to a direct sum of a normal matrix and a nilpotent matrix. We essentially derive this result from a computation with $2 \times 2$ matrices. Our key theorem has many corollaries which follow quite easily. By use of the standard inequality that the minimal eigenvalue of the real part of a given matrix is less than or equal to the real part of every eigenvalue of the matrix, we obtain applications to the case when the sequence of exponents is forcing or semiforcing. In particular, if a matrix has an infinite sequence of accretive powers where the exponents form a forcing sequence then $A$ is unitarily similar to the direct sum of a positive definite matrix and a nilpotent matrix (Theorem 3.12). Under some additional hypotheses we may conclude that the matrix is positive semidefinite (Corollary 3.9) or positive definite (Corollary 3.10). Further, if a matrix has an infinite sequence of strictly accretive powers where the exponents 
form a semiforcing sequence then $A$ is positive definite (Theorem 3.19). These results contain the theorems of [2], [6] and [9] quoted above as special cases.

In Section 4 we consider (complex) matrices $A$ such that $A^{r_{k}}$ is an $M$-matrix, $k=1,2, \ldots$, where $\left(r_{3}, r_{2}, \ldots\right)$ is an infinite semiforcing sequence and $A^{p}$ is an irreducible $Z$-matrix for some positive integer $p$ (see Hypothesis 4.2). By means of one of the corollaries in Section 3 we show that such a matrix is positively diagonally similar to a positive definite matrix (Theorem 4.9). In Section 5 we show more: A matrix satisfying the above conditions is indeed an $M M A$-matrix, viz. a matrix all of whose positive powers are irreducible $M$-matrices (Theorem 5.12). Thus we obtain an alternative proof and a generalization of Theorem 9.6 of [3], see Corollary 5.17.

We now make some observations concerning the relation of our paper and [3]. The results of that paper are not used in Sections 2, 3 and 4 of the current paper. However, Section 5 rests on the techniques and results developed in Sections 4 and 5 of [3], but the results of the other sections of [3] are not assumed here. In [3], Sections 4 and 5 contain preliminary results necessary for the development of a theory of $M M A$-matrices and related classes of matrices to be found in Section 6 of that paper. Thus, in the case of $M M A$-matrices, one could use our symmetrization results in the current paper (e.g. Corollary 5.17) to obtain an alternative development of the theory found in Section 6 of [3]. Since, in this approach, only symmetric matrices need be considered, this development would be somewhat simpler and perhaps aesthetically more appealing than the proofs in [3]. In particular, one may replace the proof of the crucial inequality in Lemma 6.8 of [3] by a standard inequality for Hermitian matrices, see our proof of Lemma 5.7.

Throughout this paper the term "positive (semi)definite matrix" will mean "positive (semi)definite Hermitian matrix".

Our definitions are numbered 2.1, 2.4, 4.1, 4.8, 5.11. See also the beginnings of various sections for notations and informal definitions.

\section{§2. Forcing sequences}

We shall use the notation $\left(r_{1}, r_{2}, \ldots\right)$ for an infinite sequence of integers and the notation $\left(r_{1}, \ldots, r_{1}\right)$ for a sequence of integers which is finite if $t$ is a positive integer and infinite if $t=\infty$. Further, "sequence of positive integers" will always mean "strictly increasing sequence of positive integers".

Definition 2.1. Let $T$ be a subset of the set $\mathbf{C}$ of all complex numbers, and let $R=\left(r_{1}, r_{2}, \ldots, r_{1}\right)$ be a (finite or infinite) sequence of positive integers. 
(i) The sequence $R$ is called a $T$-forcing sequence if for any number $c \in T$,

$$
\operatorname{Re}\left(c^{r_{k}}\right) \geqq 0, \quad k=1,2, \ldots, t
$$

implies that $c \geqq 0$.

(ii) The sequence $R$ is called a $T$-semiforcing sequence if for any (nonzero) number $c \in T$,

$$
\operatorname{Re}\left(c^{\prime k}\right)>0, \quad k=1,2, \ldots, t
$$

implies that $c>0$.

(iii) If $T=\mathbf{C}$ then a $T$-forcing [ $T$-semiforcing] sequence will simply be called a forcing [semiforcing] sequence.

Notatron 2.4. (i) We denote by $\mathbf{R}_{+}^{\prime}$ the set of all nonnegative numbers.

(ii) Let $T \subseteq \mathbf{C}, T \not \subset \mathbf{R}_{+}^{0}$. We denote

$$
\nu(T)=\inf \left\{|\arg (c)|: c \in T \backslash \mathbf{R}_{+}^{\prime \prime}\right\},
$$

where $\arg (c)$ is chosen in the half open interval $(-\pi, \pi]$.

The following sufficient conditions that a sequence is (semi)forcing are special cases of Theorems 3.4, 3.10, 3.11 and 3.14 in [5].

THEOREM 2.5. Let $R=\left(r_{1}, r_{2}, \ldots\right)$ be a sequence of positive integers with $r_{1}=1$.

(i) Assume that for every $m, m=3,4, \ldots$, either

$$
r_{m-1} \leqq 3 r_{m-2}
$$

or

$$
\frac{4 k+1}{3} r_{m-1} \leqq r_{m} \leqq(4 k+3) r_{m-2}
$$

for some positive integer $k$ (which depends on $m$ ). Then $R$ is a semiforcing sequence.

(ii) Furthermore, let $T \subseteq \mathbf{C}, T \not \subset \mathbf{R}_{+}^{\prime \prime}$, be such that $\nu(T)>0$, and suppose that $t$ is the (smallest) positive integer such that $r_{i} \nu(T) \geqq \pi / 2$. Then the sequence $\left(r_{1}, r_{2}, \ldots, r_{t+1}\right)$ is $T$-semiforcing.

(iii) Furthermore, if (2.6) is satisfied for $m=3,4, \ldots, t+1$ then the sequence $\left(r_{1}, r_{2}, \ldots, r_{t}\right)$ is $T$-semiforcing.

THEOREM 2.8. Let $R=\left(r_{1}, r_{2}, \ldots\right)$ be a sequence of positive integers with $r_{1}=1$.

(i) Assume that for every $m, m=3,4, \ldots$, either

$$
r_{m-1}<3 r_{m-2}
$$


or

$$
\frac{4 k+1}{3} r_{m-1}<r_{m}<(4 k+3) r_{m-2}
$$

for some positive integer $k$ (which depends on $m$ ). Then $R$ is a forcing sequence.

(ii) Furthermore, let $T \subseteq \mathbf{C}, T \notin \subset \mathbf{R}_{+}^{0}$, be such that $\nu(T)>0$, and suppose that $t$ is the (smallest) positive integer such that $r_{1} \nu(T)>\pi / 2$. Then the sequence $\left(r_{1}, r_{2}, \ldots, r_{t+1}\right)$ is $T$-forcing.

(iii) Furthermore, if (2.9) is satisfied for $m=3,4, \ldots, t+1$ then the sequence $\left(r_{1}, r_{2}, \ldots, r_{l}\right)$ is $T$-forcing.

We remark that in Theorems 2.5 and 2.8 the value of $k$ in (2.7) and (2.10) can only be 1 or 2, except in the case of $m=t+1$ in Part (ii) of these theorems. For a detailed discussion see Observation (3.19) in [5].

Example 2.11. The sequence $(1,3,4,8, \ldots)$ where $r_{k}=2^{k-1}, k=3,4, \ldots$, is semiforcing by Theorem 2.5 , but is not forcing since $\operatorname{Re}\left(i^{r_{k}}\right) \geqq 0$ for $k=1,2, \ldots$.

EXAmple 2.12. The sequence $(1,3,10,31,94, \ldots)$ where $r_{k}=3 r_{k-1}+1, k=$ $3,4, \ldots$, is forcing (see Corollary 3.16 in [5]).

EXAmPle 2.13. The sequence $\left(1, p, p^{2}, \ldots\right)$ where $p$ is a positive integer, $p>1$, is forcing if and only if $p=2$ and is semiforcing if and only if $p=2$ or $p=3$ (see Corollary 3.15 in [5]).

EXAmple 2.14. The sequence $(p, p+1, p+2, \ldots)$ where $p$ is a positive integer is forcing (see Corollary 3.28 in [5]).

EXAmPle 2.15. The sequence $(2,3,5,8,13, \ldots)$ where $r_{k}=r_{k-2}+r_{k-1}, k=$ $3,4, \ldots$, is not semiforcing (see the end of Section 3 in [5]).

\section{§3. Sequences of accretive powers}

If $n$ is a positive integer, we put $\langle n\rangle=\{1, \ldots, n\}$.

Let $\lambda \in \mathbf{C}$ and let $A \in \mathbf{C}^{n n}$. The index of $\lambda$ as an eigenvalue of $A$ is defined to be the order of the largest block in the Jordan canonical form of $A$ associated with $\lambda$. In particular the index of $\lambda$ as an eigenvalue of $A$ is 0 if and only if $\lambda$ is not an eigenvalue of $A$. Now let $A$ be nilpotent. Then the index (of nilpotency) of $A$ is defined to be the index of 0 as an eigenvalue of $A$. It is equal to the smallest positive integer $p$ such that $A^{p}=0$.

Let $A \in \mathbf{C}^{n n}$ and let $i, j \in\langle n\rangle$. We denote by $A[i, j]$ the $2 \times 2$ principal submatrix of $A$ based on the indices $i$ and $j$. 
We begin with our key theorem.

Theorem 3.1. Let $A \in \mathrm{C}^{n n}$ and let $\operatorname{spec}(A)$ be the multiset $\left\{\lambda_{1}, \ldots, \lambda_{n}\right\}$. Suppose that

$$
\left|\lambda_{i}\right|=\left|\lambda_{j}\right| \quad \text { implies that } \lambda_{i}=\lambda_{j}, \quad i, j \in\langle n\rangle .
$$

Let $\left(r_{1}, r_{2}, \ldots\right)$ be a (strictly increasing) infinite sequence of positive integers. If

$$
A^{r_{k}} \text { is accretive, } \quad k=1,2, \ldots,
$$

then $A$ is unitarily similar to the direct sum $H \oplus N$, where $H$ is a nonsingular normal matrix and $N$ is a nilpotent matrix of index less than or equal to $r_{1}$.

Proof. Without loss of generality we suppose that

$$
\left|\lambda_{1}\right| \geqq \cdots \geqq\left|\lambda_{h}\right|>\lambda_{h+1}=\cdots=\lambda_{n}=0 .
$$

By examination of the proof of a standard theorem due to Schur $[7$, p. 67], the matrix $A$ is unitarily similar to an upper triangular matrix $B$ with $b_{i i}=\lambda_{1}$, $i=1, \ldots, n$. Furthermore, $\operatorname{Re}\left(B^{p}\right)$ is unitarily similar to $\operatorname{Re}\left(A^{p}\right)$ for every positive integer $p$. Partition $B$ as

$$
B=\left[\begin{array}{cc}
H & C \\
0 & N
\end{array}\right],
$$

where $H$ is an $h \times h$ block. We shall show that $C=0$, that $H$ is a diagonal matrix and that $N$ is a nilpotent matrix with index less than or equal to $r_{1}$. This will prove the result.

We first show that $H$ is a diagonal matrix and that $C=0$. Suppose otherwise. Then there exist $i \in\langle h\rangle$ and $j \in\langle n\rangle$ with $i<j$ such that $b=b_{i i} \neq 0$. We choose $i, j$ satisfying the above conditions such that $j-i$ is minimal. Then

$$
b_{i k}=0, \quad i<k<j .
$$

It follows from the triangularity of $B$ and from (3.4) that for each positive integer $p$ we have

$$
\begin{aligned}
& \left(B^{p}\right)_{i i}=\lambda_{i}^{p}, \\
& \left(B^{p}\right)_{i j}=\lambda_{i}^{p},
\end{aligned}
$$

and

$$
\left(B^{p}\right)_{i j}=\sum_{q=0}^{p-1} \lambda_{i}^{p-q-1} b \lambda_{i}^{q} .
$$


Let $C_{p}=B^{p}[i, j]=B[i, j]^{p}$. In view of (3.2), we need consider only the following two cases.

Case 1. $\lambda_{i}=\lambda_{j}=\lambda$. In this case

$$
C_{p}=\left[\begin{array}{cc}
\lambda^{p} & p b \lambda^{p-1} \\
0 & \lambda^{p}
\end{array}\right]
$$

Thus

$$
\operatorname{Re}\left(C_{p}\right)=\left[\begin{array}{ll}
\operatorname{Re}\left(\lambda^{p}\right) & p b \lambda^{p-1} / 2 \\
\frac{p b \lambda^{p-1} / 2}{} & \operatorname{Re}\left(\lambda^{p}\right)
\end{array}\right] .
$$

Let $\sigma=\lambda / \lambda$. Then

$$
\operatorname{Re}\left(C_{p}\right)=\lambda^{\rho}\left[\begin{array}{ll}
\operatorname{Re}\left(\sigma^{p}\right) & p b \sigma^{p} / 2 \lambda \\
\overline{p b \sigma^{p} / 2 \lambda} & \operatorname{Re}\left(\sigma^{p}\right)
\end{array}\right] .
$$

Since $|\sigma|=1$ it now follows that for all $p$ sufficiently large the above matrix has a negative determinant. Since the sequence $r_{1}, r_{2}, \ldots$ tends to infinity, we have a contradiction to (3.3).

Case 2. $\left|\lambda_{i}\right|>\left|\lambda_{i}\right|$. In this case

$$
\begin{aligned}
C_{p} & =\left[\begin{array}{cc}
\lambda_{i}^{p} & \sum_{q=11}^{p-1} \lambda_{i}^{p-q-1} b \lambda_{i}^{q} \\
0 & \lambda_{i}^{p}
\end{array}\right] \\
& =\left[\begin{array}{cc}
\lambda^{p} & c \lambda^{p}\left(1-\nu^{p}\right) \\
0 & \lambda^{p} \nu^{p}
\end{array}\right],
\end{aligned}
$$

where $\lambda=\lambda_{i}, \nu=\lambda_{j} / \lambda_{i}$ and $c=b / \lambda(1-\nu)$. For $\sigma=\lambda /|\lambda|$ we obtain

$$
\begin{aligned}
\operatorname{Re}\left(C_{p}\right) & =\left[\begin{array}{cc}
\frac{\operatorname{Re}\left(\lambda^{p}\right)}{c \lambda^{p}\left(1-\nu^{p}\right) / 2} & \operatorname{Re}\left(\lambda^{p}\left(1-\nu^{p}\right) / 2\right.
\end{array}\right] \\
& =|\lambda|^{p}\left[\begin{array}{cc}
\frac{\operatorname{Re}\left(\sigma^{p}\right)}{c \sigma^{p}\left(1-\nu^{p}\right) / 2} & c \sigma^{p}\left(1-\nu^{p}\right) / 2 \\
\operatorname{Re}\left(\sigma^{p} \nu^{p}\right)
\end{array}\right] .
\end{aligned}
$$

Since $|\sigma|=1$ and $|\nu|<1$ it now follows that for all $p$ sufficiently large the above matrix has a negative determinant. As in Case 1 this contradicts (3.3).

Hence, the matrix $H$ is diagonal and $C=0$.

We now show that $N$ is nilpotent of index less than or equal to $r_{1}$. Since $N$ is strictly upper triangular it is clear that $N$ is nilpotent. Suppose that $N^{r_{1}} \neq 0$. Then 
$b=\left(N^{\prime \prime}\right)_{i j} \neq 0$ for some $i, j \in\langle n\rangle, i<j$. Hence

$$
\operatorname{Re}\left(N^{r_{1}}[i, j]\right)=\left[\begin{array}{cc}
0 & b / 2 \\
b / 2 & 0
\end{array}\right]
$$

which is not positive semidefinite, contrary to (3.3). Hence the index of $N$ is less than or equal to $r_{1}$.

The hypothesis that $\left(r_{1}, r_{2}, \ldots\right)$ is infinite cannot be omitted from Theorem 3.1 as demonstrated by the matrix in Example 3.15 below.

We have a number of corollaries which are immediate.

Corollary 3.5. Let $A \in \mathbf{C}^{n n}$ and suppose that the index of 0 as an eigenvalue of $A$ is at most 1. If $A$ satisfies the hypotheses of Theorem 3.1 then $A$ is normal.

Proof. Since the matrix $H \oplus N$ in the conclusion of Theorem 3.1 has the same Jordan canonical form as $A$, the nilpotent matrix $N$ is either absent or is of index 1 and hence $N=0$. It follows that $A$ is unitarily similar to a normal matrix and thus $A$ is normal.

COROLlary 3.6. Let $\left(r_{1}, r_{2}, \ldots\right)$ be an infinite sequence with $r_{1}=1$. If $A \in \mathbf{C}^{n n}$ satisfies the hypotheses of Theorem 3.1 then $A$ is normal.

Proof. In this case, the nilpotent matrix $N$ in the conclusion of Theorem 3.1 is either absent or is a zero matrix and the result follows.

Condition (3.2) cannot be omitted from the hypotheses of Theorem 3.1 or Corollaries 3.5 and 3.6 as is shown by the following example.

EXAMPLE 3.7. Let

$$
A=\left[\begin{array}{cc}
1 & 1 \\
0 & e^{\pi i / 3}
\end{array}\right]
$$

Then $\operatorname{Re}(A)>0$ and $A^{5 k}=I, k=1,2, \ldots$ and hence (3.3) holds for $r_{1}=1$, $r_{k}=6(k-1), k=2,3, \ldots$. But $A$ is not normal, and hence, being nonsingular, $A$ cannot be unitarily similar to the direct sum of a normal and a nilpotent matrix.

Corollary 3.8. Let $A \in \mathrm{C}^{n n}$ and let $\left(r_{1}, r_{2}, \ldots\right)$ be an infinite sequence of positive integers such that (3.3) holds. If the eigenvalues of $A$ are nonnegative then the matrix $A$ is unitarily similar to the direct sum of a positive definite matrix and $a$ nilpotent matrix of index less than or equal to $r_{1}$.

Proof. Since the eigenvalues of $A$ are nonnegative, condition (3.2) is 
satisfied. Hence, since a nonsingular normal matrix with nonnegative eigenvalues is positive definite the result follows from Theorem 3.1.

COROLlary 3.9. Let $A \in \mathbf{C}^{n n}$ and let $\left(r_{1}, r_{2}, \ldots\right)$ be an infinite sequence of positive integers such that (3.3) holds. If the eigenvalues of $A$ are nonnegative and the index of 0 as an eigenvalue of $A$ is at most 1 , then $A$ is positive semidefinite.

COROLlary 3.10. Let $A \in \mathbf{C}^{n n}$ and let $\left(r_{1}, r_{2}, \ldots\right)$ be an infinite sequence of positive integers such that (3.3) holds. If the eigenvalues of $A$ are positive then $A$ is positive definite.

It is now easy to prove further results under the assumption that the sequence $\left(r_{1}, r_{2}, \ldots\right)$ is forcing or semiforcing.

The following lemma is very well known, e.g. 5.2 .7 in [7, p. 169].

Lemma 3.11. Let $A \in \mathbf{C}^{n n}$ and let $\lambda \in \operatorname{spec}(A)$. Then $\operatorname{Re}(\lambda)$ is greater than or equal to the minimal eigenvalue of $\operatorname{Re}(A)$.

THEOREM 3.12. Let $A \in \mathbf{C}^{n n}$ and let $T$ be a subset of $\mathbf{C}$ which contains $\operatorname{spec}(A)$. Suppose that $\left(r_{1}, r_{2}, \ldots\right)$ is an increasing infinite $T$-forcing sequence. If (3.3) holds then the matrix $A$ is unitarily similar to the direct sum of a positve definite matrix and a nilpotent matrix of index less than or equal to $r_{1}$.

Proof. Let $\lambda \in \operatorname{spec}(A)$. By Lemma 3.11 we see that (3.3) implies that

$$
\operatorname{Re}\left(\lambda^{r_{k}}\right) \geqq 0, \quad k=1,2, \ldots
$$

Since $\left(r_{1}, r_{2}, \ldots\right)$ is a $T$-forcing sequence, it follows that $\lambda \geqq 0$. Thus the result follows immediately from Corollary 3.8 .

Theorem 3.12 has corollaries analogous to Corollaries 3.5 and 3.6. We state the second of these explicitly as Corollary 3.14. This corollary generalizes the results of [2] and [9] in the finite dimensional case since $r_{k}=r$ and $r_{k}=2^{k-1}$, $k=1,2, \ldots$, are forcing sequences.

Corollary 3.14. Let $A \in \mathbf{C}^{n n}$ and let $T$ be a subset of $\mathrm{C}$ which contains $\operatorname{spec}(A)$. Suppose that $\left(r_{1}, r_{2}, \ldots\right)$ is an infinite $T$-forcing sequence with $r_{1}=1$. If (3.3) holds then the matrix $A$ is positive semidefinite.

Theorem 3.12 and Corollary 3.14 do not hold in general for finite $T$-forcing sequences as demonstrated by the following example.

EXAMPLE 3.15. Let

$$
A=\left[\begin{array}{ll}
1 & 1 \\
0 & 1
\end{array}\right]
$$


Let $T=\{1\}$. Clearly every sequence is $T$-forcing. In particular we may choose the sequence (1). Observe that $A$ is accretive, so (3.3) is satisfied. However the conclusion sof Theorem 3.12 or Corollary 3.14 do not hold.

We also remark that the condition that the sequence $\left(r_{1}, r_{2}, \ldots\right)$ is $T$-forcing cannot be omitted from Theorem 3.12 and Corollary 3.14. In fact, for every sequence $\left(r_{1}, r_{2}, \ldots\right)$ which is not $T$-forcing one can find a matrix $A$ whose spectrum is contained in $T$ such that $A$ satisfies (3.3) but not the conclusion of Theorem 3.12. For proof note that by Definition 2.1 there exists a complex number $\lambda \in T$ which is not nonnegative and which satisfies

$$
\operatorname{Re}\left(\lambda_{i}^{r_{k}}\right) \geqq 0, \quad k=1,2, \ldots
$$

The matrix $\lambda I \in \mathbf{C}^{n n}$ furnishes an example.

We now impose conditions which permit us to conclude that $A$ is positive definite.

COROLlary 3.16. Let $A \in \mathbf{C}^{n n}$ be a nonsingular matrix and let $T$ be a subset of $C$ that contains $\operatorname{spec}(A)$. Suppose that $\left(r_{1}, r_{2}, \ldots\right)$ is an infinite $T$-forcing sequence. If (3.3) holds then the matrix $A$ is positive definite.

Corollary 3.17. Let $A \in \mathbf{C}^{n n}$ and let $T$ be a subset of $\mathbf{C}$ that contains $\operatorname{spec}(A)$. Suppose that $\left(r_{1}, r_{2}, \ldots\right)$ is an infinite $T$-forcing sequence. If (3.3) holds and there exists a positive integer $p$ such that $A^{p}$ is strictly accretive then $A$ is positive definite.

Proof. Let $\lambda \in \operatorname{spec}(A)$. If $A^{p}$ is strictly accretive then it follows from Lemma 3.11 that $\operatorname{Re}\left(\lambda^{p}\right)>0$ and hence $A$ is nonsingular. The result now follows from Corollary 3.16 .

Since $(1,2,3, \ldots)$ is a forcing sequence, Corollary 3.17 generalizes Johnson's result in [6].

Theorem 3.12 and Corollaries 3.16 and 3.17 do not hold if "forcing" is replaced by "semiforcing". Consider the following example.

EXAMPLE 3.18. Let $r_{k}=3^{k-1}, k=1,2, \ldots$ This sequence is semiforcing by Lemma 2.12. Consider the $1 \times 1$ matrix

$$
A=\left[e^{\pi / \hbar}\right] .
$$

Then $\operatorname{Re}\left(A^{r_{1}}\right)>0$, and $\operatorname{Re}\left(A^{r_{k}}\right) \geqq 0, k=2,3, \ldots$. Thus $A$ satisfies the hypotheses of Theorem 3.12 and its corollaries (with $T=\mathbf{C}$ ) but the conclusions of these results are obviously false for $A$. 
However we have the following result for semiforcing sequences.

THEOREM 3.19. Let $A \in \mathbf{C}^{n n}$ and let $T$ be a subset of $\mathbf{C}$ that contains $\operatorname{spec}(A)$. Suppose that $\left(r_{1}, r_{2}, \ldots\right)$ is an infinite $T$-semiforcing sequence. Suppose that

$$
A^{r_{k}} \text { is strictly accretive, } \quad k=1,2, \ldots
$$

Then $A$ is positive definite.

Proof. Let $\lambda \in \operatorname{spec}(A)$. Then it follows from Lemma 3.11 and (3.20) that

$$
\operatorname{Re}\left(\lambda^{r_{k}}\right)>0, \quad k=1,2, \ldots
$$

Since $\left(r_{1}, r_{2}, \ldots\right)$ is a semiforcing sequence, it follows from (3.21) that $\lambda>0$. The result now follows from Corollary 3.10 .

Theorem 3.19 does not hold in general for finite $T$-semiforcing sequences as demonstrated by Example 3.15.

Note that the condition that the sequence $\left(r_{1}, r_{2}, \ldots\right)$ is $T$-semiforcing cannot be omitted from Theorem 3.19; see a similar remark about Theorem 3.12 after Example 3.15.

For specific semiforcing sequences stronger results may hold. Let $\left(r_{1}, r_{2}, \ldots\right)$ be the semifrocing sequence $(1,3,4,8,16, \ldots)$ considered in Section 2 . If $c$ is a complex number such that $\operatorname{Re}\left(c^{r_{k}}\right) \geqq 0$ for $k=1,2, \ldots$ then either $c$ is nonnegative or $c$ is pure imaginary. Hence for this sequence (3.3) implies that $A$ is unitarily similar to the direct sum of a positive definite matrix, a skew-Hermitian matrix and a nilpotent matrix of index less than or equal to $r_{1}$. Further, if (3.3) holds and either $A$ or $A^{3}$ is strictly accretive then $A$ is positive definite. For proof observe that in this case it follows from Lemma 3.11 that $A$ cannot have pure imaginary eigenvalues.

Since one can easily find a normal matrix $A$ satisfying (3.2) which is not accretive, it follows that the natural converses of Theorem 3.1 and Corollaries 3.5 and 3.6 do not hold. On the other hand, converses of all subsequent theorems and corollaries in this section are true and trivial.

Let $A \in \mathbf{R}^{n n}$ and suppose that $A$ has real eigenvalues. Then $A$ is orthogonally similar to a real upper triangular matrix. Hence, repeating the proof of Theorem 3.1 in this case, we see that for such matrices we may replace "unitarily similar" by "orthogonally similar", "normal matrix" by "real symmetric matrix", "positive (semi)definite matrix" by "positive (semi)definite real matrix" in Theorem 3.1 and Corollaries 3.5 and 3.6. Consequently, the same replacements may be made in Theorems 3.11 and 3.17 and Corollaries 3.14 and 3.15 for real $A$ (without any assumptions on the location of $\operatorname{spec}(A)$ ). 


\section{§4. Applications to sequences of $M$-matrix powers}

DefinItION 4.1. Let $A \in \mathbf{R}^{n n}$.

(i) Then $A$ is called a $Z$-matrix if $a_{i j} \leqq 0, i \neq j, i, j=1, \ldots, n$.

(ii) Further, $A$ is called an $M$-matrix if $A$ is a $Z$-matrix and all eigenvalues of $A$ have nonnegative real part.

In this section we mainly discuss matrices satisfying the following hypothesis.

Hypothesis 4.2. The matrix $A$ belongs to $\mathbf{C}^{n n}$. Assume that there exist a subset $T$ of $\mathbf{C}$, a sequence $R=\left(r_{1}, r_{2}, \ldots\right)$ and a positive integer $p$ satisfying the following conditions:

$R$ is an infinite increasing $T$-semiforcing sequence,

and

$$
\begin{gathered}
\operatorname{spec}(A) \subseteq T, \\
A^{\prime k} \text { is an } M \text {-matrix, } \quad k=1,2, \ldots,
\end{gathered}
$$

LEMmA 4.7. Let the matrix A satisfy Hypothesis 4.2. Then all eigenvalues of $A$ are nonnegative. Further, if $\nu$ is the minimal real eigenvalue of $A$ then it is simple and the associated right and left eigenvectors may be chosen (entrywise) positive.

Proof. Let $\lambda \in \operatorname{spec}(A)$, where $\lambda \neq 0$. Since every nonzero eigenvalue of an $M$-matrix has a positive real part (e.g. [1, p. 150]), it follows from (4.5) that $\operatorname{Re}\left(\lambda^{r_{k}}\right)>0, k=1,2, \ldots$. It now follows from (4.3) and (4.4) that $\lambda>0$. Hence all eigenvalues of $A$ are nonnegative.

Now let $\nu$ be the minimal eigenvalue of $A$. Observe that the minimal eigenvalue of $A^{p}$ is $\nu^{p}$. Since (4.6) holds, $\nu^{p}$ is a simple eigenvalue and the associated right and left eigenvectors $x$ and $y^{\top}$ respectively may be chosen positive. Therefore $\nu$ is a simple eigenvalue of $A$ and by standard arguments using the Jordan canonical form we deduce that $x$ and $y^{\top}$ are the associated eigenvectors of $A$.

Definitron 4.8. (i) Let $D \in \mathbf{C}^{n n}$. Then $D$ is called a positive diagonal matrix if $D$ is a diagonal matrix and all diagonal entries of $D$ are positive.

(ii) Let $A, B \in C^{n n}$. Then $A$ and $B$ are called positively diagonally similar if there exists a positive diagonal matrix $D$ in $C^{n \pi}$ such that $B=D^{-1} A D$. 
(iii) Let $A, B \in \mathrm{C}^{n n}$. Then $A$ and $B$ are called cogredient if there exists a permutation matrix $P$ in $C^{n n}$ such that $B=P^{\mathrm{T}} A P$.

Evidently positive diagonal similarity is an equivalence relation on $\mathbf{C}^{n n}$.

We now partially generalize Theorem 9.6 of [3] in the case that the matrix $A$ has a sequence of $M$-matrix powers. As observed in the introduction, our proof is independent of the proof in [3].

THEOREM 4.9. Let the matrix A satisfy Hypothesis 4.2. Then $A$ is positively diagonally similar to a positive semidefinite matrix.

Proof. By Lemma 4.7 we may let $X$ and $Y$ be positive diagonal matrices such that $X e$ and $e^{\mathrm{T}} Y$ are the right and left eigenvectors of $A$ associated with the nonnegative (minimal) eigenvalue $\nu$, where $e=[1,1, \ldots, 1]^{\mathrm{T}}$. Let $D=$ $\left(X Y^{-1}\right)^{1 / 2}$, let $B=D^{-1} A D$ and let $u=(X Y)^{1 / 2} e$. Observe that $u$ and $u^{\mathrm{T}}$ are the right and left eigenvectors of $B$ associated with $\nu$. Thus

$$
\operatorname{Re}\left(B^{r_{k}}\right) u=\nu^{r_{n}} u \geqq 0, \quad k=1,2, \ldots
$$

Clearly, $\operatorname{Re}\left(B^{r_{k}}\right)$ is a $Z$-matrix. Since $u$ is positive, it follows from (4.10) by a very well known result (e.g. [8, Theorem 4]) that $\operatorname{Re}\left(B^{r_{k}}\right)$ is an $M$-matrix and thus positive semidefinite. By Lemma 4.7 all eigenvalues of $B$ are nonnegative. Furthermore, the minimal eigenvalue of $B$ is simple and hence the index of 0 as an eigenvalue of $B$ is at most 1 . By Corollary 3.9 the matrix $B$ is positive semidefinite.

We now prove some results that are needed in Section 5 for a generalization of Theorem 6.12 of [3] in the case that the matrix $A$ has a sequence of $M$-matrix powers.

Let $A$ be a Hermitian matrix. As is well known, e.g. [4, p. 156], $A$ may be written in the form

$$
A=\lambda_{1} E_{1}+\cdots+\lambda_{4} E_{4},
$$

where $\lambda_{1}, \ldots, \lambda_{4}$ are the distinct (real) eigenvalues of $A$ and $E_{1}, \ldots, E_{4}$ are mutually orthogonal idempotent Hermitian matrices whose sum is the identity matrix. The form (4.11) is called the spectral decomposition of $A$. If $A$ further satisfies Hypothesis 4.2 then in view of Lemma 4.7 we may assume that

$$
0 \leqq \lambda_{1}<\cdots<\lambda_{q}
$$

LEMMA 4.13. Let $A$ be a Hermitian matrix that satisfies Hypothesis 4.2 and let the spectral decomposition of $A$ be given by (4.11) where (4.12) holds. Then 
$E_{1}>0$. Furthermore, if $n>1$ then $q>1$ and $E_{q}$ is cogredient to a direct sum of $m$ singular irreducible (symmetric idempotent) $M$-matrices, where $m<n$.

Proof. As is well known the idempotent $E_{1}=x y^{*}$, where $x$ and $y^{*}$ are the right and left eigenvectors of $A$ associated with $\lambda_{1}$ under the normalization $y^{*} x=1$. By Lemma 4.7 we have

$$
E_{1}>0
$$

Suppose that $q=1$. Then $A$ is a scalar matrix and it follows from (4.6) that $n=1$. Thus we have shown that $n>1$ implies that $q>1$.

Suppose that $n>1$. It follows easily from (4.12) that

$$
\lim _{k \rightarrow x}\left(A / \lambda_{q}\right)^{r_{k}}=E_{q} .
$$

Hence, by (4.5), $E_{q}$ is an $M$-matrix. Since $A$ is Hermitian, $E_{q}$ is symmetric and idempotent and therefore cogredient to a direct sum of $m$ irreducible symmetric idempotent $M$-matrices. Since $q>1$, we have $E_{1} E_{q}=0$, and it follows from (4.14) that each direct summand in $E_{q}$ is singular. Since $E_{q} \neq 0$, it obviously follows that $m<n$.

\section{\$5. Further applications to sequences of $M$-matrix powers}

Henceforth we shall use the results of Sections 4 and 5 of [3], but not the results of other sections of that paper.

Let $C \in \mathbf{C}^{\prime \prime \prime \prime}$ and let $U \in \mathbf{C}^{n n}$, where the rows and columns of $U$ are partitioned into subsets of cardinality $n_{1}, \ldots, n_{m}$ with $n_{1}+\cdots+n_{m}=n$. In Definition 4.1 of [3] we introduced the inflation matrix $C \times x U$. Thus if $\mathrm{C}\left(n_{1}, \ldots, n_{m}\right)$ consists of the set of matrices $U \in \mathbf{C}^{n n}$ partitioned as

$$
U=\left[\begin{array}{cccc}
U_{11} & U_{12} & \cdots & U_{1 m} \\
\cdot & \cdot & \cdots & \cdot \\
\cdot & \cdot & \cdots & \cdot \\
\cdot & \cdot & \cdots & \cdot \\
U_{m 1} & U_{m 2} & \cdots & U_{m m}
\end{array}\right]
$$

where $U_{i i}$ is $n_{i} \times n_{i}, i=1, \ldots, m$, then

$$
C \times x U=\left[\begin{array}{cccc}
c_{11} U_{11} & c_{12} U_{12} & \cdots & c_{1 m} U_{1 m} \\
\cdot & \cdot & \cdots & \cdot \\
\cdot & \cdot & \cdots & \cdot \\
\cdot & \cdot & \cdots & \cdot \\
c_{m 1} U_{m 1} & c_{m 2} U_{m 2} & \cdots & c_{m m} U_{m m}
\end{array}\right] .
$$


The operation of inflation generalizes the Hadamard and Kronecker products. Given a partition $\left(n_{1}, \ldots, n_{m}\right)$ of $n$, we introduce in Definition 4.3 of [3] a certain subclass $U\left(n_{1}, \ldots, n_{m}\right)$ of partitioned $n \times n$ matrices of rank 1 with no zero entries. Thus $U \in \mathbf{C}^{n n}$ belongs to $U\left(n_{1}, \ldots, n_{m}\right)$ if and only if there exist $u_{i}, v_{i} \in \mathbf{C}^{n_{i}}$ such that $u_{i}, v_{i}$ have no zero components and

$$
\begin{gathered}
\left(v_{i}\right)^{\mathrm{T}} u_{i}=1, \quad i=1, \ldots, m, \\
U_{i j}=u_{i}\left(v_{j}\right)^{\mathrm{T}}, \quad i, j=1, \ldots, m .
\end{gathered}
$$

Here we need consider only positive $U$. The set of positive $U$ belonging to some class $U\left(n_{1}, \ldots, n_{m}\right)$ is here denoted by $\mathscr{U}_{m n}$. If $U \in \mathcal{U}_{m n}$, then $C x x U$ has interesting properties, see Section 4 of [3]. We require three additional simple lemmas.

Let $U \in \mathcal{U}_{m n}$. As in [3] we write

$$
G(U)=I_{n}-\left(I_{m} \times x U\right)
$$

Then $G(U)$ is the direct sum of $m$ irreducible idempotents of nullity 1 , see Section 4 of [3]. Further, since $U>0, G(U)$ is an $M$-matrix, see Section 5 of [3].

Lemma 5.4. Let $A \in \mathbf{C}^{n n}, C \in \mathbf{C}^{m m}$. Let $U$ be a symmetric matrix in $\mathscr{U}_{m n}$ and let $\lambda$ be a real number. If

$$
A=C \mathrm{xx} U+\lambda G(U)
$$

where $G(U)$ is given by (5.3), then $A$ is a Hermitian matrix [real symmetric matrix] if and only if $C$ is a Hermitian matrix [real symmetric matrix].

Proof. We give the proof in the Hermitian case. Suppose $A$ is Hermitian. By (5.3) the matrix $G(U)$ is real symmetric. Thus $C x x U$ is Hermitian and since $U$ has no 0 elements it follows from (5.2) that $C$ is Hermitian. The converse is equally easy.

Lemma 5.6. Let $A \in \mathbf{C}^{n n}, C \in \mathbf{C}^{m m}, U \in \mathcal{U}_{m n}$ and $\lambda \in \mathbf{C}$. Suppose that (5.5) holds. Then

(i) $\operatorname{spec}(C) \subseteq \operatorname{spec}(A)$.

(ii) If $A$ is a $Z$-matrix then $C$ is a $Z$-matrix.

(iii) If $A$ is an $M$-matrix then $C$ is an $M$-matrix.

(iv) If $A$ is irreducible then $C$ is irreducible.

Proof. (i) This is contained in Corollary 4.22 of [3]. 
(ii) Since $U>0$ and in view of (5.2), if $C$ has a positive off-diagonal element then the corresponding block of $A$ is positive.

(iii) Follows immediately from (i) and (ii).

(iv) In view of (5.2), if $C$ has a zero off-diagonal element then the corresponding block of $A$ is zero.

Under additional hypotheses we may obtain stronger results. Proofs are provided for the implications not covered by the corresponding parts of Lemma 5.6 .

LEMMA 5.7. Let $C$ be a Hermitian matrix in $\mathbf{C}^{n n}$. Let $U$ be a symmetric matrix in $u_{m n}$, where $m<n$, and let $\lambda$ be a real number such that $\lambda>\nu$, where $\nu$ is the maximal eigenvalue of $C$. If (5.5) holds then

(i) $\operatorname{spec}(A)=\operatorname{spec}(C) \cup\{\lambda\}$.

(ii) $A$ is a $Z$-matrix if and only if $C$ is a $Z$-matrix.

(iii) $A$ is an $M$-matrix if and only if $C$ is an $M$-matrix.

(iv) $A$ is irreducible if and only if $C$ is irreducible.

Proof. (i) This is again contained in Corollary 4.22 of [3].

(ii) Let $C$ be a $Z$-matrix. Since $C$ is Hermitian, it follows from the Cauchy interlacing inequalities, e.g. [7, p. 119], that

$$
c_{i i} \leqq \nu, \quad i=1, \ldots, m .
$$

Since $\nu<\lambda$, we have

$$
c_{i i}<\lambda, \quad i=1, \ldots, m .
$$

Thus, if $A$ and $I=I_{n}$ are partitioned in the same way as $U$, it follows that

$$
A_{i i}=c_{i i} U_{i i}+\lambda(I-U)_{i i}=\lambda I_{i i}-\left(\lambda-c_{i i}\right) U_{i i}, \quad i=1, \ldots, m .
$$

Since $U_{i i}>0$ it follows from (5.9) that $A_{i i}$ is a $Z$-matrix, $i=1, \ldots, m$. Further, since $C$ is a $Z$-matrix, we have $c_{i j} \leqq 0, i \neq j$. Hence, since $U_{i j}>0$, it follows from

$$
A_{i j}=c_{i j} U_{i j,} \quad i \neq j, \quad i, j=1, \ldots, m,
$$

that $A_{i j} \leqq 0, i \neq j, i, j=1, \ldots, m$. Thus $A$ is a $Z$-matrix.

(iii) Follows immediately from (i) and (ii).

(iv) If $m=1$ then $A=A_{i i}$ and the irreducibility of $A$ follows from (5.9) and (5.10).

Now let $m>1$ and let $C$ be irreducible. Since $c_{i j} \neq 0$ implies that $c_{i j} U_{i j} \neq 0$, $i \neq j, i, j=1, \ldots, m$, the irreducibility of $\boldsymbol{A}$ follows from the irreducibility of $C$. 
The following definition may be found in Definition 1.1 of [3].

Definition 5.11. Let $A \in \mathbf{C}^{n n}$. Then $A$ is called an $M M A$-matrix if $A^{\prime}$ is an irreducible $M$-matrix for $t=1,2, \ldots$.

THEOREM 5.12. Let the matrix $A$ satisfy Hypothesis 4.2. Then $A$ is an MMA-matrix.

Proof. By Theorem 4.9 we may assume, without loss of generality, that $A$ is a positive semidefinite matrix, since the class of $M$-matrices is invariant under positive diagonal similarity. We prove our theorem by induction on $n$. If $n=1$ then the result is an immediate consequence of (4.3), (4.4) and Definition 2.1. Assume that the result holds if $n<s$, where $s>1$, and let $n=s$. Let the spectral decomposition of $A$ be given by (4.11) where (4.12) holds. Let $B=A-\lambda_{q} E_{q}$. By Lemma 4.13 above and by Lemma 5.5 of [3] there exists a positive matrix $U \in \mathcal{U}_{m n}$ such that $E_{q}=G(U)$, where $m$ is the number of irreducible direct summands in $E_{q}$ and where $G(U)$ is given by (5.3). Further, by Lemma 4.13 above we have $m<n$. Since $E_{q} B=0=B E_{q}$, it follows from Lémma 4.23 in [3] that there is a matrix $C \in \mathcal{U}_{m n}$, such that $B=C x x U$.

We shall now show that $C$ satisfies Hypothesis 4.2 (with $n$ replaced by $m$ ). Since $G(U) B=0=B G(U)$, it follows from (4.12) in [3] that

$$
A^{\prime}=\left(C^{\prime} x x U\right)+\lambda^{\prime} G(U), \quad t=1,2, \ldots
$$

By Lemma 5.6(i) and (4.4) we now have

$$
\operatorname{spec}(C) \subseteq \operatorname{spec}(A) \subseteq T .
$$

By Lemma 5.6(iii), (4.5) and (5.13) imply that

$$
C^{r_{k}} \text { is an } M \text {-matrix, } \quad k=1,2, \ldots
$$

By Parts (ii) and (iv) of Lemma 5.6, (4.6) and (5.13) imply that

$$
C^{p} \text { is an irreducible } Z \text {-matrix. }
$$

Therefore, by (4.3) and (5.14)-(5.16), the matrix $C$ satisfies Hypothesis 4.2. By the inductive assumption $C$ is an $M M A$-matrix and, by Lemma 5.4, the matrix $C$ is symmetric. It follows that $C^{\prime}$ is a symmetric irreducible $M$-matrix, $q=1,2, \ldots$. Hence, by (5.13) and by Lemma 5.7(iii) with $C, \lambda$ and $A$ replaced respectively by $C^{\prime}, \lambda^{\prime}$ and $A^{\prime}, t=1,2, \ldots$, it follows that $A$ is an $M M A$-matrix.

Of course, an $M M A$-matrix satisfies Hypothesis 4.2, and a matrix positively diagonally similar to an $M M A$-matrix is an $M M A$-matrix. Thus we may 
combine Theorems 4.9 and 5.12 to obtain the following corollary which generalizes Theorem 9.6 of [3].

Corollary 5.17. Let $A \in \mathrm{C}^{n n}$. Then the following are equivalent.

(i) A satisfies Hypothesis 4.2 .

(ii) $A$ is positively diagonally similar to a symmetric MMA-matrix.

(iii) $A$ is an MMA-matrix.

\section{ACKNOWLEDGEMENT}

We acknowledge with thanks some helpful remarks and additional references by $\mathrm{S}$. Friedland.

\section{REFERENCES}

1. A. Berman and R. J. Plemmons. Nonnegative Matrices in the Mathematical Sciences, Academic Press 1979.

2. C. R. DePrima and B. K. Richard. A characterization of the positive cone of $B(h)$, Indiana Univ. Math. J. 23 (197.3). 16.3-172.

3. S. Friedland. D. Hershkowitz and H. Schneider, Matrices whose powers are M-matrices or $Z$-matrices, to appear.

4. P. R. Halmos. Finite-Dimensional Vector Spaces, 2nd edn., Van Nostrand, 1958.

5. D. Hershkowitz and H. Schneider, Sequences, wedges and associated sets of complex numbers, to appear.

6. C. R. Johnson. Powers of matrices with positive definite real part. Proc. Amer. Math. Soc. 50 (1975). 85-91.

7. M. Marcus and H. Minc. A Survey of Matrix Theory and Matrix Inequalities, Allyn and Bacon. 1964

8. H. Schneider. An inequality for latent roots applied to determinants with dominant principal diagonal, J. London Math. Soc. 28 (1953). 13-20.

9. E. S. Shiu, Growth of numerical ranges of powers of Hilbert space operators, Michigan Math. J. 23 (1976), 155-160.

10. B. Sz.-Nagy and C. Foias, Harmonic analysis of operators on Hilbert space, American Elsevier, 1970. 